\title{
Headwave Stacking in Terms of Partial Derivative Wavefield
}

\author{
Changsoo Shin \\ School of Civil, Urban and Geosystem Engineering, Seoul National University, San 56-1, \\ Sinlim-Dong, Kwanak-Gu, Seoul, 151-742, Korea
}

\begin{abstract}
Head wave stacking and velocity analysis are used to image the shallow subsurface, while CMP stacking and velocity analysis are used to image deep structures of the earth. I relate these concepts to partial derivative seismograms, which gives stacking straight line of head waves. The stacking straight line can be described kinematically by partial derivative seismograms, resulting in an interesting seismic imaging relationship.
\end{abstract}

Key words: Head wave, Velocity analysis, Partial derivative seismogram, Head wave stacking, image.

\section{INTRODUCTION}

Since imaging subsurface has been an important tool to geophysicists, there have been extensive studies about imaging the subsurface. Some of the studies are based on the fact that kinematics of a scattered or a Born perturbation wavefield can be represented by the sum of travel time from a source to a receiver through a scatterer (Apostoiu-Marin and Ehinger, 1997; Bleistein et al., 1985; Gardner et al., 1974). Geophysicists have imaged the subsurface by summing seismic signals along a hyperbola representing the kinematics of the Born perturbation seismogram for any source and receiver configuration. Shin and Chung (1999) showed that the CMP stacking hyperbola, obtained in velocity analysis, is kinematically expressed by partial derivative seismograms with respect to the coordinate of the interface separating the geological medium.

In a similar way to summing reflection hyperbola in the prestack migration, Landa et al. (1995) obtained shallow subsurface image by displaying the summed signal of headwaves at the intercept time. Landa et al. (1995) showed that stacking headwaves along a straight line

Received February 13, 2004; Accepted October 4, 2004 Published October 31, 2004

*Corresponding Author: Changsoo Shin

E-mail: css@model.snu.ac.kr

Address: School of Civil, Urban and Geosystem Engineering, Seoul National Univ., S. Korea, San 56-1, Sillim-dong, Gwanak-gu, Seoul, 151-742, Korea. determined by computing semblance for refracted waves (at common shot gathers) produced images for the shallow subsurface.

In this paper, we will show that the straight line, used by Landa et al. (1995) for stacking headwaves, is kinematically represented by the partial derivative seismogram obtained when we perturb velocity and density at the interface coordinate of a shallow subsurface. We begin with reviewing the partial derivative seismogram outlined by Pratt et al. (1998) and exploited by Shin and Chung (1999) to evaluate the CMP stacking hyperbola in terms of the partial derivative wavefield. Next, we evaluate if we can express the straight line used in stacking headwaves as the partial derivative seismogram kinematically by calculating and displaying the partial derivative seismograms for a given model. This is followed with mathematical expressions of stacking seismogram. By showing numerical examples, we confirm that the headwave-stacking technique started by Landa et al. (1995) is also a useful tool to image the shallow subsurface.

\section{PARTIAL DERIVATIVE SEISMOGRAM}

Pratt et al. (1998) and Shin (1988) showed how to calculate a partial derivative seismogram of the elastic and the scalar wave equations in the frequency domain. We modify this approach to be applicable in the time domain as Shin and Chung (1999) did. The scalar wave equation is given as

$$
\frac{\partial}{\partial x}\left(\frac{1}{\rho} \frac{\partial d}{\partial x}\right)+\frac{\partial}{\partial z}\left(\frac{1}{\rho} \frac{\partial d}{\partial z}\right)-\frac{1}{k} \frac{\partial^{2} d}{\partial t^{2}}=f(x, z, t)
$$

where $x$ and $z$ are the horizontal and vertical distances, $t$ is the time, $d(x, z, t)$ is the seismic data, $\rho(x, z)$ is the density, $k(x, z)$ is the bulk modulus and $f(x, z, t)$ is the source function. The discretized form of the scalar wave equations can be obtained by a finite-difference or a finite-element approach as

$$
\mathbf{M d}+\mathbf{K d}=\mathbf{f}
$$

where $\mathbf{M}$ is the mass matrix, $\mathbf{K}$ is the stiffness matrix, $\mathbf{f}$ is the source vector, and $\mathbf{d}$ is the discretized seismic data vector. 
At the finite-difference grid set shown in Fig. 1, if we identify a velocity and a density at a node $i$ as $v_{i}$ and $\rho_{i}$, we can define our model parameter vector, $\mathbf{p}$, to be simply $\mathbf{p}=\left[\rho_{1}, v_{1}, \rho_{2}, v_{2}, \cdots, \rho_{n}, v_{n}\right]^{T}$. By taking the partial derivative of equation (2) with respect to density or velocity at the interface coordinate and then by rearranging the order of the derivatives to preserve the original discretized wave equation, we can obtain

$$
\mathbf{M} \frac{\partial \ddot{\mathbf{d}}}{\partial \mathbf{p}}+\mathbf{K} \frac{\partial \mathbf{d}}{\partial \mathbf{p}}=\mathbf{f}^{*}
$$

where $(\partial \mathbf{d} / \partial \mathbf{p})$ is the partial derivative seismogram and $\mathbf{f}^{*}$ is the virtual source vector defined as

$$
\mathbf{f}^{*}=-\frac{\partial \mathbf{M}}{\partial \mathbf{p}} \ddot{\mathbf{d}}-\frac{\partial \mathbf{K}}{\partial \mathbf{p}}=\mathbf{f}^{*}
$$

To synthesize a partial derivative seismogram recorded at the surface for a source located at the top of the grid set in Fig. 1, we use equation (3). In order to solve equation (3), we need the virtual source $\mathbf{f}^{*}$ in addition to the forward wavefield at each grid point. The partial derivative seismograms at the surface nodes computed by the virtual sources make up a huge Jacobian matrix $\mathbf{J}$. A component of Jacobian matrix $J_{i j}$ corresponds to the partial derivative wavefield due to the change of velocity or density at the $j$ th node for the $i$ th source gather. In Fig. 3, we display the virtual sources required to perturb the velocities or densities at the $k$ th, $l$ th, and $m$ th nodal point in Fig. 2. The physical properties of the three layers in Fig. 2 are shown in Table 1. Figs. $4 a, 4 b$, and $4 c$ show the partial derivative seismograms computed by using the virtual sources in Fig. 3. We note that the first arrival events of

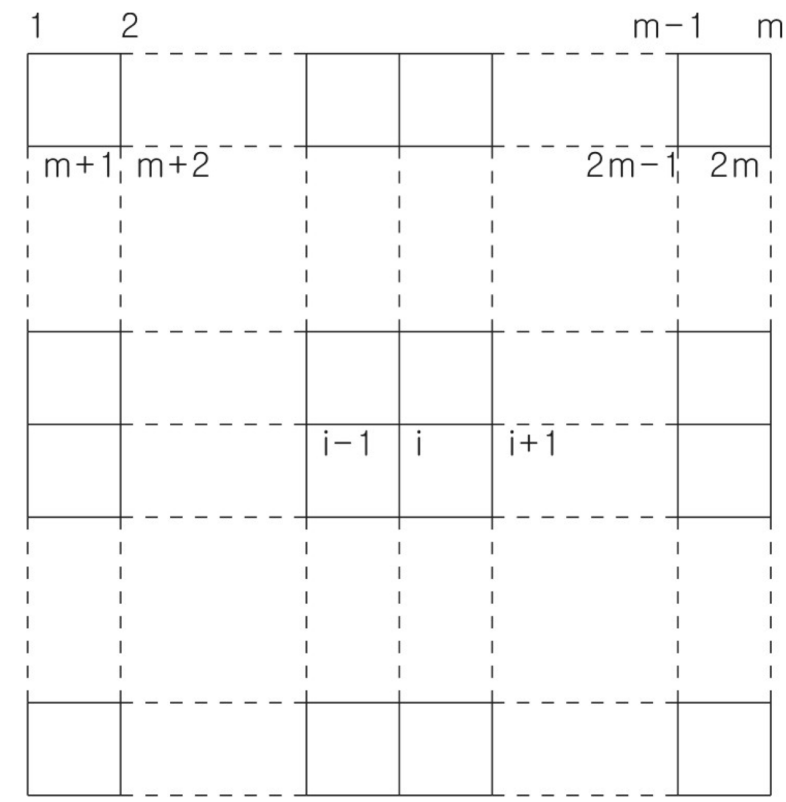

Fig. 1. A finite-difference grid set.

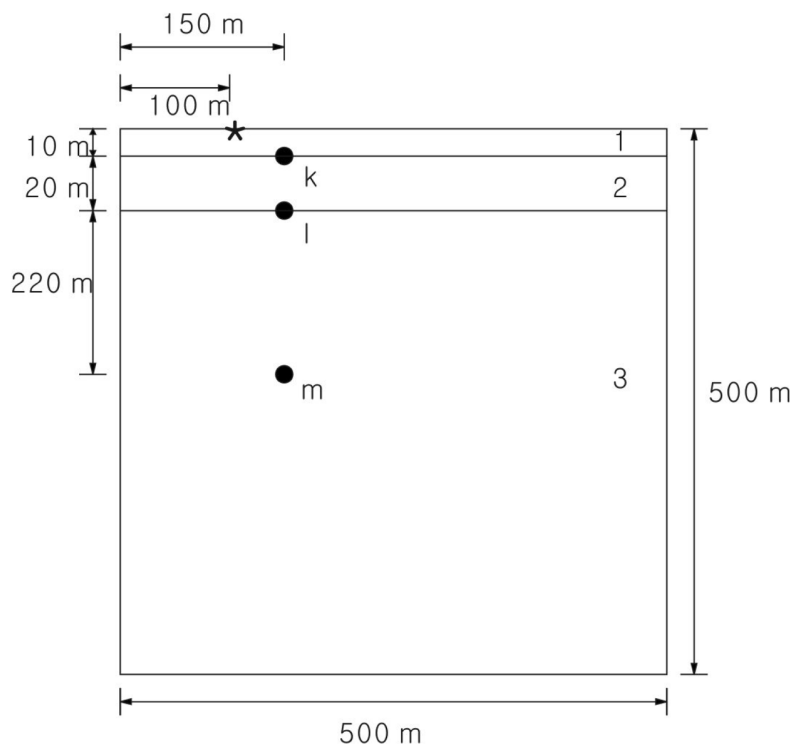

Fig. 2. A geological model used in computing the partial derivative wavefield with respect to velocity or density. The symbol * denotes the source used in generating the forward seismogram. The symbols, and indicate the depth points where velocity or density are perturbed for computing the partial derivative seismogram. The physical properties of the three layers are shown in Table 1.

Tables 1. The physical properties of the three layers in Fig. 2

\begin{tabular}{ccc}
\hline Layer & Velocity & Density \\
\hline 1 & $1000 \mathrm{~m} / \mathrm{s}$ & $1.0 \mathrm{~g} /$ \\
2 & $2000 \mathrm{~m} / \mathrm{s}$ & $2.0 \mathrm{~g} /$ \\
3 & $3000 \mathrm{~m} / \mathrm{s}$ & $3.0 \mathrm{~g} /$ \\
\hline
\end{tabular}

the partial derivative seismogram at the shallow $k$ th and th nodal point resemble a straight line or can be approximated as a straight line (e.g., Figs. 4a and 4b); those at the deeper $m$ th nodal point change into a hyperbola (see Fig. 4c). From these Figures, we demonstrate that the first arrival events of the partial derivative seismogram generated by the virtual sources located in the shallow subsurface can be represented as a straight line.

\section{MATHEMATICS OF STACKING SEISMOGRAM}

How much the field seismogram is responsive to a certain interface segment can be estimated by taking a zero-lag crosscorrelation between the field seismogram and the partial derivative seismogram with respect to material parameter such as velocity or density at the interface (Shin and Chung, 1999). By measuring and displaying the zero-lag crosscorrelation at each nodal point, one can image the subsurface structure (Shin and Chung, 1999). This technique has often been used in prestack Kirchhoff 

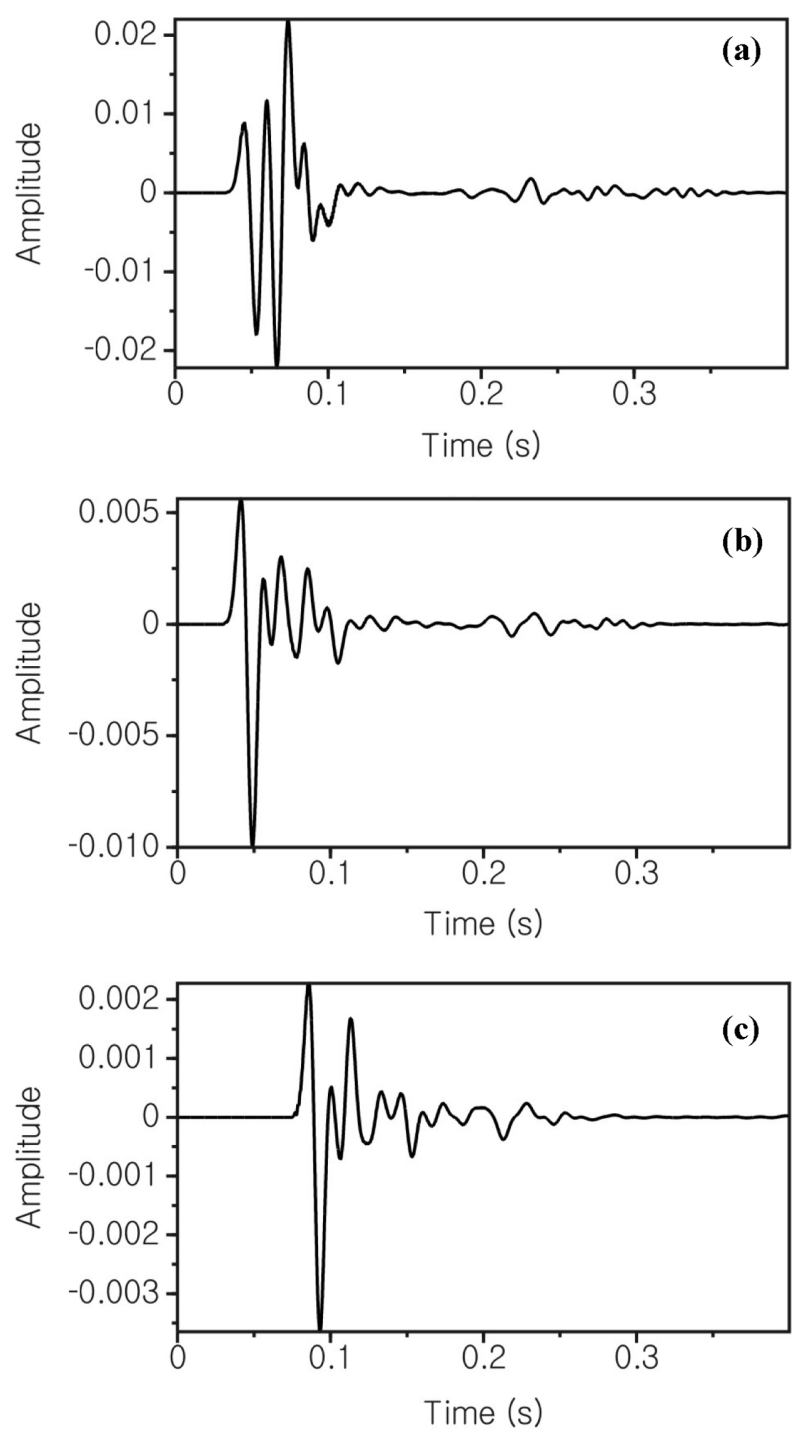

Fig. 3. Virtual sources calculated by a finite-difference method when velocities or densities are perturbed at the (a) th, (b) th and (c) th grid point shown in Fig. 2.

depth migration. The zero-lag crosscorrelation of field seismogram $\mathbf{d}$ and the partial derivative seismogram $\mathbf{J}$ is expressed by

$$
\mathbf{r}=\mathbf{J}^{T} \mathbf{d}
$$

where the Jacobian matrix $\mathbf{J}$, approximated kinematically by the asymptotic ray theory in the prestack Kirchhoff depth migration, could be calculated via equation (3). We recognize the vector $\mathbf{r}$ as an unscaled image or stacked value of the measured seismic data. Since the partial derivative seismogram in Fig. 4c have different amplitudes and different phases along the hyperbola (Shin and Chung, 1999), we can identify the hyperbola of the

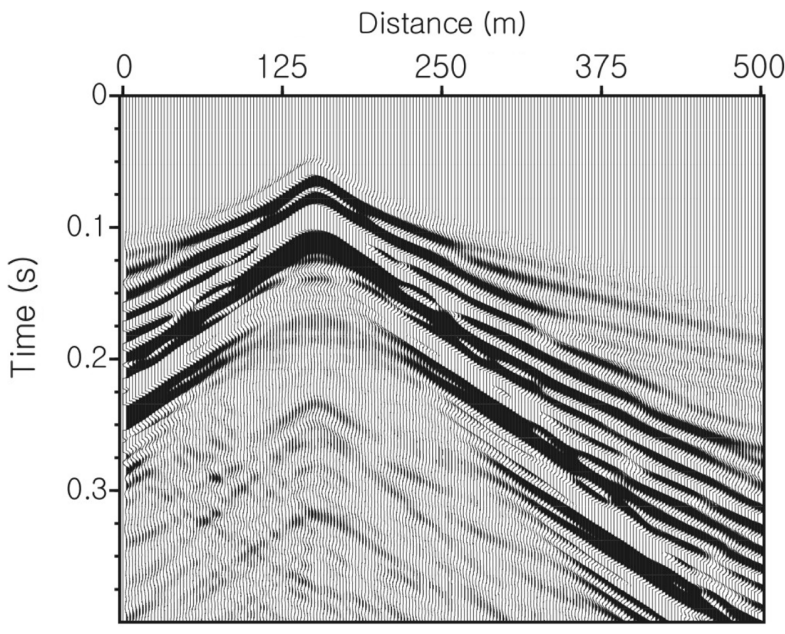

(a)

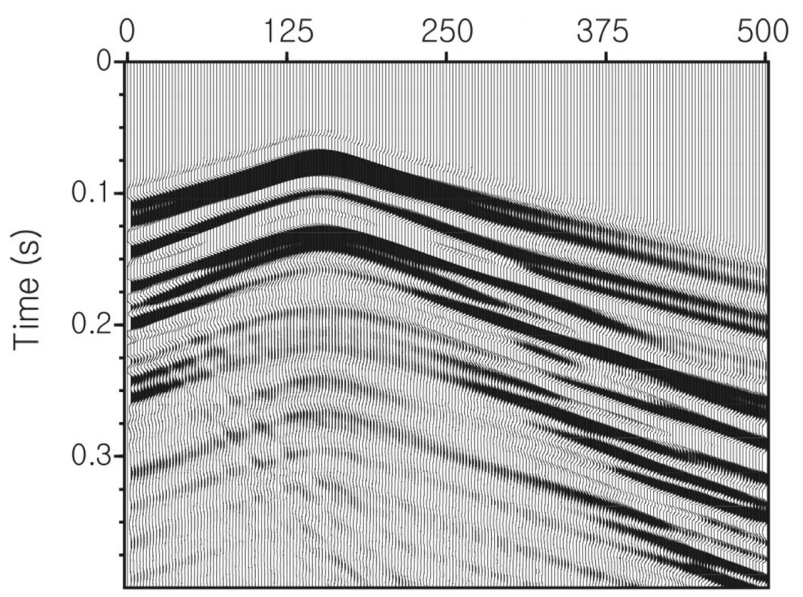

(b)

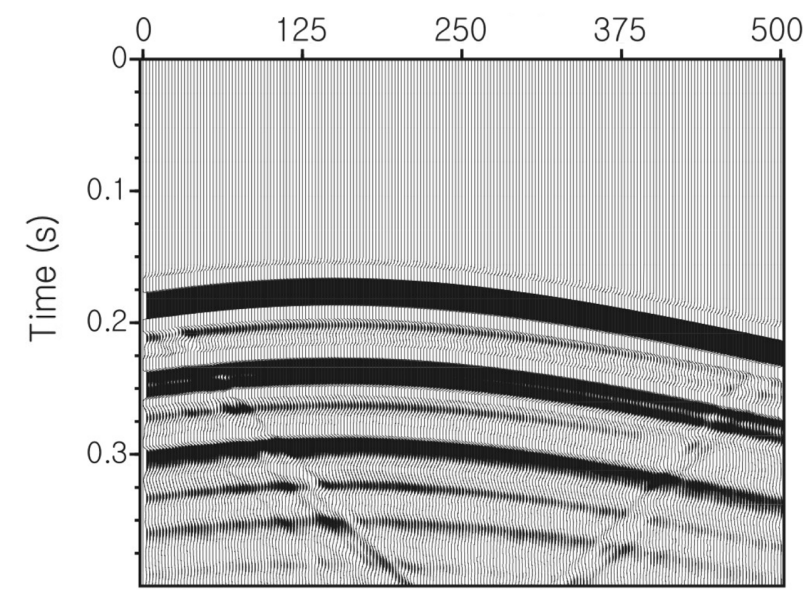

(c)

Fig. 4. Partial derivative seismograms computed by finite-difference method for the virtual source at the (a) th, (b) th and (c) th nodal point shown in Fig. 3.

partial derivative seismogram in equation (5) as a weighted 
Kirchhoff hyperbola. Equation (5) can be written in an integral form as

$$
r_{i}=\int_{x_{\min }}^{x_{\max }} \int_{0}^{t_{\max }} \frac{\partial u(x, t)}{\partial p_{i}} d(x, t) d x d t
$$

where $r_{i}$ is the th element of the unscaled image vector $\mathbf{r},\left(\partial u(x, t) \partial p_{i}\right)$ is the partial derivative seismogram with respect to the velocity or the density of the $i$ th nodal point in the subsurface, $x$ is the horizontal offset, $t$ is the time and $d(x, t)$ is the field seismogram.

In the usual prestack Kirchhoff migration, the kinematics of the partial derivative seismogram is only considered. If we consider a summing wavefield along the hyperbola representing the kinematics of the partial derivative seismogram shown in Fig. 4c, stacking seismograms can be represented as

$$
r_{i}=\int_{x_{\min }}^{x_{\max }} \int_{0}^{t_{\max }} d(x, t) \delta\left(t-\sqrt{\left(t_{0}^{2}+\frac{x^{2}}{v^{2}}\right)}\right) d x d t
$$

where $t_{0}$ is the minimum travel time from a source to a receiver through a scatterer, $v$ is the arbitrary velocity which is yet to be determined and $\delta$ is the delta function. In the prestack Kirchhoff migration, we estimate the kinematics of the partial derivative seismogram using a ray tracing technique.

We already showed that the first arrival events of the partial derivative seismogram shown in Fig. $4 \mathrm{a}$ and $4 \mathrm{~b}$ can be approximated as a straight line. An integral expression for stacking the headwaves along the straight line (corresponding to the kinematics of the partial derivative seismogram) is given as

$$
r_{i}=\int_{x_{\min }}^{x_{\max }} \int_{0}^{t_{\max }} d(x, t) \delta\left(t-t_{0}-x / v_{s}\right) d x d t
$$

where $r_{i}$ is the $i$ th element of the unscaled image vector $\mathbf{r}, d(x, t)$ is the field seismogram, $\delta$ is the delta function, $x$ is the horizontal offset, $t$ is the time, $t_{0}$ is the intercept time, and $v_{s}$ is the stacking velocity (for refracted waves) where we have the maximum velocity spectrum. The $\delta$ function expresses the kinematics of the partial derivative seismogram with respect to the velocity or the density at the $i$ th nodal point in the shallow subsurface. In order to obtain the stacking velocity for the refracted waves, Landa et al. (1995) performed a velocity analysis as in the conventional reflection data processing. The velocity analysis applied by Landa et al. (1995) is different from that of conventional reflection data processing in measuring the semblance not along the trial hyperbolas but along the trial straight lines.

\section{NUMERICAL EXAMPLES}

We stacked field seimograms along straight lines which approximate partial derivative seismogram kinematically for a horizontal layer and a dipping layer model. We also carried out velocity analysis along a number of straight lines for obtaining stacking velocity as Landa et al. (1995) did.

Fig. 5 shows the horizontal model composed of two horizontal layers whose velocities are 1000 and $3000 \mathrm{~m} / \mathrm{s}$. The velocity spectrum calculated for selecting the optimal stacking velocity is displayed in Fig. 6. Since the velocity spectrum is obtained by computing coherence along a number of straight lines for each time window, it is natural that the velocity spectrum has larger coherence when $v=1000 \mathrm{~m} / \mathrm{s}$ at $t=0$ (for the direct wave) and when $v=$ $1000 \mathrm{~m} / \mathrm{s}$ at the intercept time, as shown in Fig. 6. The subsurface image results from stacking the synthetic seismogram with the optimal stacking velocities. Fig. 7 shows the subsurface image for the horizontal model.

As a second numerical example, we take the dipping layer model depicted in Fig. 8. Figs. 9 and 10 show the velocity spectrum and the stacked image obtained for the dipping layer model. In Fig. 9, we can see that larger

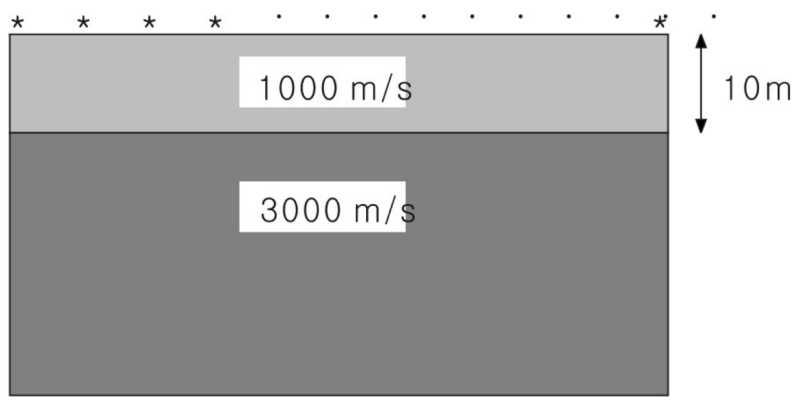

Fig. 5. A horizontal layer model.

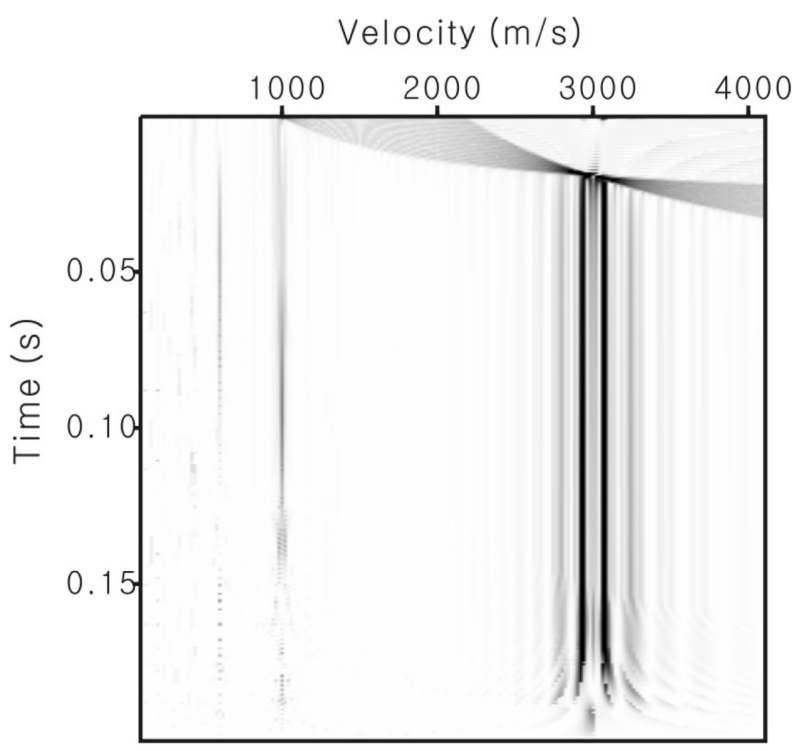

Fig. 6. The velocity spectrum obtained for the horizontal layer model in Fig. 5. 


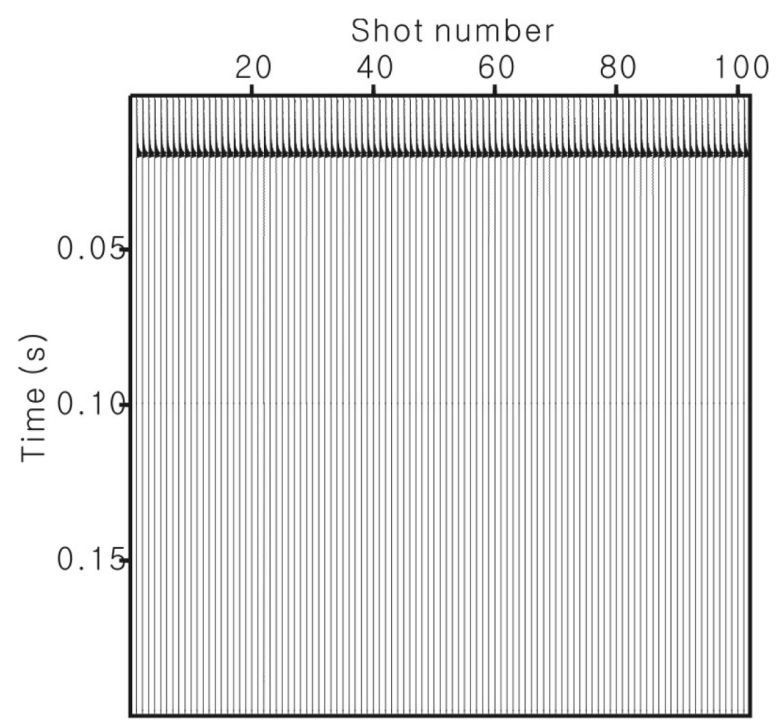

Fig. 7. The subsurface image obtained by stacking the refracted waves along the straight lines (which are interpreted as the kinematics of the partial derivative seismogram) at the synthetic common shot gathers for the dipping layer model.

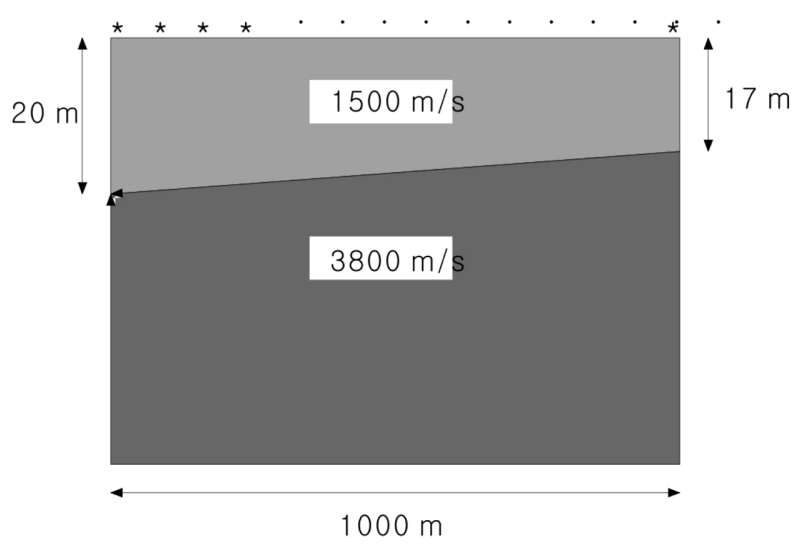

Fig. 8. A dipping layer model.

coherence appear when $v=1500 \mathrm{~m} / \mathrm{s}$ at $t=0$ and when $v=3800 \mathrm{~m} / \mathrm{s}$ at the intercept time. These velocities correspond to the first and second layer velocities. The stacked image in Fig. 10 is compatible with the dipping layer model in Fig. 8.

\section{CONCLUSION}

By analyzing the kinematics of the partial derivative seismograms, we showed that the straight line used by Landa et al. (1995) for the headwave-stacking technique is a substitution for the stacking hyperbola of the reflection seismogram in the prestack Kirchhoff migration. Our

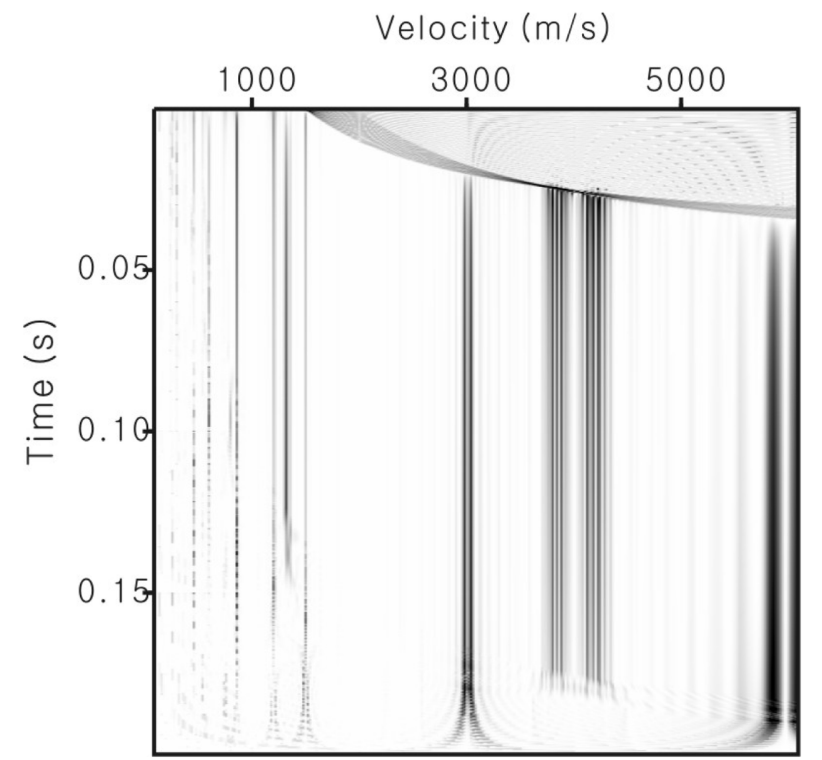

Fig. 9. The velocity spectrum obtained for the dipping layer model in Fig. 8.

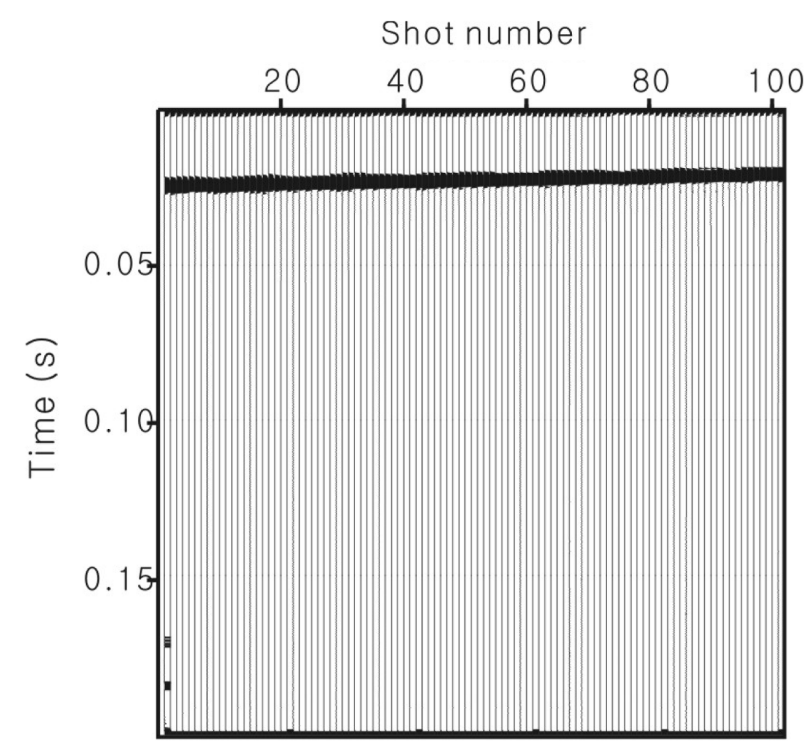

Fig. 10. The subsurface image obtained by stacking the refracted waves along the straight lines (which are interpreted as the kinematics of the partial derivative seismogram) at the synthetic common shot gathers for the dipping layer model.

analysis indicates that the straight line used in stacking the refracted waves is the kinematics of partial derivative seismogram with respect to the velocity or the density at a depth point located in the shallow subsurface. As shown by Landa et al. (1995), the headwave-stacking method can be used to stack or migrate the shallow subsurface seismic data. 


\section{REFERENCES}

Apostoiu-Marin, I. and Ehinger, A., 1997, Kinematic interpretation in the prestack depth-migrated domain, Geophysics, 62, p. 1226-1237.

Bleistein, N. and Gray, S. H., 1985, An extension of the Born inversion method to a depth dependent reference profile, Geophys. Prosp., 33, p. 999-1022.

Gardner, G. H. F., French, W. S., and Matzuk, T., 1974, Elements of migration and velocity Analysis, Geophysics, 39, p. 811-825.

Landa, E., Keydar, S., and Kravtsov, A., 1995, Determination of a shallow velocity-depth model from seismic refraction data by coherence inversion, Geophys. Prosp., 43, p. 177-190.

Pratt, R. G., Shin, C., and Hicks, J. G., 1998, Gauss-Newton and full Newton method in frequency-space seismic waveform inversion, Geophys. J. Int., 133, p. 341-362.

Shin, C., 1988, Nonlinear elastic wave inversion by blocky parameterization: $\mathrm{Ph}$. D. thesis, Univ. of Tulsa.

Shin, C. and Chung, S., 1999, Understanding CMP stacking hyperbola in terms of partial derivative wavefield, Geophysics, 64, p. 678-700.
Changsoo Shin received a $\mathrm{PhD}$ (1988) in geophysics from the University of Tulsa. After working at the Korea Institute of Geology, Mining, and Materials from 1990 to 1996, he became an associate professor of geophysics at Seoul National University. He has led the national research laboratory of seismic inversion and imaging of Seoul National University since June 2000. His interests are the numerical modeling of wave propagation, full waveform inversion, and geophysical imaging. (E-mail: css@model.snu.ac.kr)

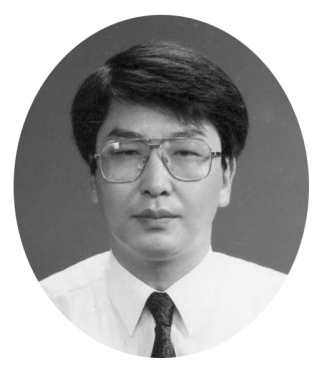

Changsoo Shin 